\title{
Research on the realization of interactive dynamic system based on Flash ActionScript
}

\author{
Fu Jie \\ School of Primary Education, Zaozhuang University, China
}

\begin{abstract}
*43287266@qq.com
system interface development, Flash game development and so on.

Keywords: Flash ActionScript; interactive dynamic system; programming; courseware making

Through this study, we have learned Flash ActionScript3.0 language in a comprehensive and in-depth way, and mastered application skills of the language on the basis of basic knowledge and grammars. Finally, we can use the language flexibly in daily practices, especially of the application and practices in interactive dynamic systems.
\end{abstract}

\begin{abstract}
Digital signature plays a more and more important role in e-commerce. The basic methods of digital signature are introduced and a digital signature scheme based on digital certificate is proposed in this paper. Digital certificate is generated after personal information is written. The effective data, RSA parameters and signature results are included in digital certificate. The related RSA parameters of private key certificate are encrypted by private key password. The digital digest is gotten through hash algorithm in this scheme. Digital signature is operated by using RSA parameters in private key certificate and the signature is verified by RSA parameters in public key certificate by verifier.
\end{abstract}

\section{Introduction}

Flash, an excellent vector drawing and animation software, inherits all advantages of vector graphics software, so it can produce animation with outstanding sound and color as well as high interactivity. In particular, it is able to produce animation files with very small size and is very flexible in network which has high requirements on speed, so it is very popular in network and animation industry. ActionScript, a ECMAScript-based and object-oriented programming language, not only creates wizard and interactive elements, but also expands Flash and thereby displaying strong interactivity of Flash, so users can not only watch the animation, but also participate in the animation. Therefore, users are able to determine the process and results of an event through clicking, selection and other actions as Flash has advantages in interactive technology.

With the progress of science and technology, new versions of Flash have been constantly launched, such as ActionScript 1.0 at the beginning and ActionScript Flash ActionScript3.0 at the time being, all of which introduce new elements and add some keywords, objects, methods and other language elements. At present, Flash is mainly used in the field of mobile phone development, teaching application, site construction, software

\section{Introduction of Flash ActionScript3.0 language}

As a programming language specifically used in runtime environment of Flash Player, ActionScript3.0 has a variety of functions such as human-computer interaction, data exchange, etc. ActionScript 3.0 is essentially different from the previous version of ActionScript, and it is a powerful and object-oriented programming language which meets industry standards. As a milestone in the history of Flash programming languages, ActionScript3.0 is an important language for developing application programs (RIA). ActionScript 3, a powerful object-oriented programming language, marks an important stage in the evolution of Flash Player Runtime.

FLASH combined with ActionScript is widely used in the design of courseware which are vivid with high interactivity and excellent demonstration effect of contents, so it has become the preferred tool of most frontline teachers for making courseware. ActionScript3.0 have been applied in many fields, and will certainly be applied in more extensive fields in today's era of object-oriented programming.

ActionScript 3 consists of two parts such as core language and flash player API. Core language is used to define the structure of a programming language, such as declarations, representations, conditions, loops and types. Flash player API is composed of a series of precisely defined flashplayer functions. ActionScript3.0 has a new feature of mining remaining performance of a computer. The rule representation supports XML which enhances operation performance. ECMAScript for XML (E4X) makes XML to be a common data type, greatly simplifying the processing of XML. New DisplayListAPI will coordinate virtual objects, and normalized DOM event model improves the representation and response of 
objects. Of course, these are just partial new experiences of ActionScript3.0.

\section{Design and realization of interactive dynamic system of Flash ActionScript}

Taking flash dynamic interactive courseware the Motion of the Earth and Geographical Significance as the example, and the procedures of the design and realization of ActionScript language-based interactive dynamic system are as follows:

\subsection{Programming environment of ActionScript}

"Action panel" is a tool to write ActionScript scripts provided by flash to users. Single clicking the "window" | "action" command or pressing F9 can open "actions panel". The actions panel consists of four parts:

Script element list area.ActionScript elements were grouped based on different categories, and selected elements were inserted into script pane by double click or direct drag.

Actions refer to action statements inserted in the script. Operators contain those that can be used in a statement. Functions contain those that can be used in a statement. Constants contain those that can be used in a statement. Properties contain those that can be used in a statement. Object contain those that can be used in the script and their properties, events, and methods.

Flash UI components contain Flash UI (user interface) components which can be used in the script and their properties, events and a list of methods.

Script editing area.ActionScript code can be entered into this pane.

Parameter setting area.Toolbar has a variety of auxiliary functions of writing codes, which can simplify the process of coding. There are two ways to write ActionScript Codes such as expert mode and standard mode, and coding mode can be switched by script assistant.

\subsection{Time axis control function}

Time axis control functions, mainly used to control the play, stop and skip of frames and scenes, are divided into the following types:

Play ( ) ; stop ( ) ; gotoAndPlay ( ) ; gotoAndStop ( ) ; nextFrame ( ) ; prevFrame ( ) ; nextScene ( ) ; prevScene ( ) ; stopAllSounds ( ) ;

\subsection{Button events.}

onDragOut $=$ function ()\{\}

Note: in the case that users click mouse button on the button and then drag the mouse pointer outside the button
OnDragOver $=$ function ()\{\}

Note: in the case that users press mouse button outside the button, and then drag the mouse pointer over the button.

OnKeyDown $=$ function ()\{\}

Note: in the case that the button has keyboard focus and a key is pressed.

OnKeyUp $=$ function ()\{\}

Note: in the case that the button has input focus and a key is released.

OnKillFocus $=$ function (newFocus:Object) \{\}

Note: in the case that the button loses keyboard focus.

OnPress $=$ function ()\{\}

Note: in the case that the button is pressed.

OnRelease $=$ function ()\{\}

Note: in the case that the button is released.

OnReleaseOutside $=$ function ()\{\}

Note: in this case that the button is pressed when mouse pointer is located into the button, and then the mouse pointer is moved outside the button and the mouse button is released.

OnRollOut $=$ function ()\{\}

Note: in the case that the mouse pointer is moved outside button area.

OnRollOver $=$ function ()\{\}

Note: in the case that the mouse pointer is moved over button area.

OnSetFocus = function (oldFocus:Object) \{\}

Note: in the case that the button receives keyboard focus.

\section{Typical codes which realize interactive functions:}

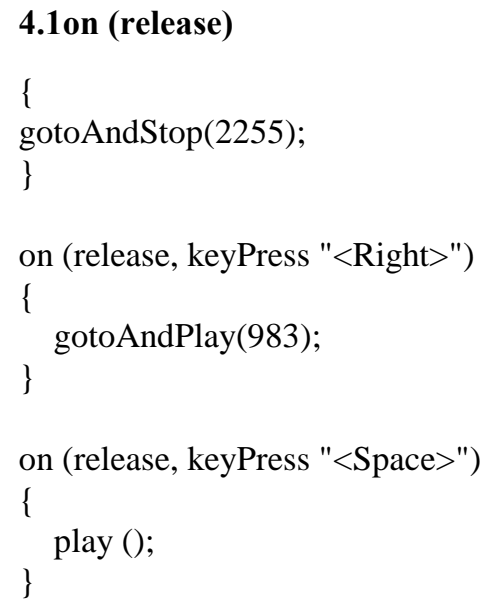




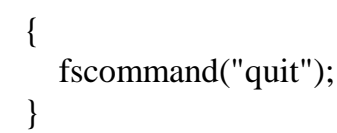

\section{2 on (release)}

\{

tellTarget("_root.showme")

\{

gotoAndPlay(2);

tellTarget("")

\{

\} // End of TellTarget

\} // End of TellTarget

\}

\section{3 function create(i_name)}

\{

this.attachMovie("ball",i_name,2);

\}

create("ball1");

duplicateMovieClip("ball1","ball2",3);

setProperty("ball2",_x,600);

\section{Conclusions}

The paper studies the detailed contents and examples of ActionScript3.0 language learning, and the realization process of interactive courseware and interactive dynamic systems, which helps people who are interested in learning the language in the study and relevant applications of the language, and help them to master the application skills of the language on the basis of basic knowledge and grammars, especially of the application and practices in interactive dynamic systems.

\section{References}

[1] Porath. Pure and Applied Chemistry.Some recently developed fractionation procedures and their application to peptide and protein hormones[J]. J. 2013(3).

[2] Jinlong Wang,Qianchuan Zhao,Haitao Li. A Multi-agent Based Evaluation Framework and Its Applications[J]. IEEE/CAA Journal of Automatica Sinica. 2014(02).

[3] Fei TANG,Rui ZHANG,Hongda LI. Attribute-based non-interactive key exchange[J]. Science China(Information Sciences). 2017(01).

[4] Somsri Wiwanitkit,Beuy Joob,Viroj Wiwanitkit. Cerebral malaria: An interactive brain mapping study[J]. Asian Pacific Journal of Tropical Medicine. 2016(08).

[5] Kuo Kuang Fan,Ko Jung Lin.KINECT with FLASH ActionScript 3.0 Applications in Interactive Design[J]. Applied Mechanics and Materials. 2012(145). 